\title{
Médiévales
}

Langues, Textes, Histoire

\section{Un trompe-l'œil maléfique : l'image du sabbat dans les manuscrits enluminés de la cour de Bourgogne (à propos du Traité du crisme de Vauderie de Jean Taincture, vers 1460-1470)}

\section{Franck Mercier}

\section{(2) OpenEdition}

\section{Journals}

Édition électronique

URL : https://journals.openedition.org/medievales/931

DOI : 10.4000/medievales.931

ISSN : 1777-5892

Éditeur

Presses universitaires de Vincennes

Édition imprimée

Date de publication : 1 juin 2003

Pagination : $97-116$

ISBN : 2-84292-142-9

ISSN : 0751-2708

Référence électronique

Franck Mercier, « Un trompe-l'œil maléfique : l'image du sabbat dans les manuscrits enluminés de la cour de Bourgogne (à propos du Traité du crisme de Vauderie de Jean Taincture, vers 1460-1470) », Médiévales [En ligne], 44 I printemps 2003, mis en ligne le 02 novembre 2010, consulté le 23 avril 2022 URL : http://journals.openedition.org/medievales/931; DOI : https://doi.org/10.4000/medievales.931

Ce document a été généré automatiquement le 23 avril 2022

Tous droits réservés 


\section{Un trompe-l'œil maléfique : l'image du sabbat dans les manuscrits enluminés de la cour de Bourgogne (à propos du Traité du crisme de Vauderie de Jean Taincture, vers 1460-1470)}

Franck Mercier

\section{NOTE DE L'ÉDITEUR}

Les figures auxquelles réfère ce document sont présentes dans la version imprimée du numéro 44.

1 L'imaginaire du sabbat est d'abord un imaginaire du livre, et plus précisément encore de la culture écrite et savante. Participant de l'indicible, et par là-même peut-être infigurable, le sabbat est rarement représenté au $\mathrm{xv}^{\mathrm{e}}$ siècle. De fait, pour cette époque, nous en conservons peu d'images. Les premiers éléments figurés susceptibles de s'y rattacher apparaissent vers le milieu du $\mathrm{Xv}^{\mathrm{e}}$ siècle sous une forme encore fragmentaire, à l'instar de ces petites "vaudoises passe-martin » qui pointent le bout de leur balai dans les marges d'un manuscrit du Champion des Dames de Martin le Franc ${ }^{1}$. Il n'est pas sans intérêt de relever que ce manuscrit fut copié et enluminé à Arras, vers 1451, avant de rejoindre la collection du duc de Bourgogne, Philippe le Bon, puisque c'est encore dans le contexte de la cour bourguignonne - une cour amoureuse du faste et des images - que surgissent, quelques années plus tard, entre 1460 et 1470, les premières représentations iconographiques complètes du sabbat des sorciers et des sorcières. 
Fig. 1 - Martin Le Franc, Le Champion des Dames. (Ms. fr. 12476, fo $105 v^{\circ}$. Cliché BnF, Paris, D.R.)

2 Il s'agit d'une courte série de trois miniatures placées chacune en frontispice de trois luxueux manuscrits de la traduction française du Tractatus contra sectam vaudensium (ou Traité du crisme de Vauderie) de Jean Taincture ${ }^{2}$. Il est difficile de préciser les conditions exactes de la commande de ces manuscrits de prestige ${ }^{3}$. On sait seulement que ces copies furent réalisées par des ateliers flamands pour le compte de hauts dignitaires de la cour de Bourgogne. Un inventaire de 1467 signale la présence de l'un d'eux dans la bibliothèque ducale. Un autre encore fut acheté par Louis de Bruges, seigneur de la Gruthuse, l'un des personnages les plus considérables de l'entourage de Philippe le Bon ${ }^{4}$.

Pour la première fois peut-être, le sabbat, que l'on rencontre jusque-là surtout dans les textes des spécialistes de la répression ou dans les aveux extorqués aux accusés, accède au stade de la représentation, dans le domaine bien particulier de la peinture sur livre. Et l'on voit par là que l'image du sabbat demeure encore inséparable de l'écrit.

Le Traité du crisme de Vauderie de Jean Taincture occupe une place à part dans l'ensemble de la littérature savante consacrée à la sorcellerie démoniaque ${ }^{5}$. Ce n'est pas l'œuvre d'un juriste ou d'un inquisiteur mais celle d'un éminent théologien, gradué de l'Université de Cologne, principalement connu pour son éloquence sacrée ainsi que ses commentaires sur Thomas $\mathrm{d}^{\prime}$ Aquin ${ }^{6}$. Il rédige son traité à Tournai en 1460 dans un contexte assez agité, marqué par le développement en Artois d'une véritable chasse aux sorciers et aux sorcières restée célèbre sous le nom de Vauderie d'Arras ${ }^{7}$.

Le traité se compose de deux parties bien distinctes. Dans la première, l'auteur s'efforce de dire l'ampleur du crime par le moyen d'une comparaison avec l'idolâtrie, l'hérésie et l'infidélité mahométane. Elle se prolonge par une longue exhortation à l'adresse des prélats, des juges mais surtout des princes. Tous sont appelés à lutter énergiquement contre le nouveau fléau des sorciers. La seconde partie de l'ouvrage, plus théorique, est principalement consacrée à l'étude des mécanismes de l'illusion diabolique. En traduisant lui-même son traité en français, Jean Taincture visait probablement à atteindre le public privilégié des princes et des grands seigneurs laïcs. La cour de Bourgogne paraît la plus réceptive à son message comme l'atteste la provenance des manuscrits de luxe parvenus jusqu'à nous.

\section{Une scène emblématique de la démonolâtrie}

6 L'étude de cette brève série iconographique révèle l'existence d'un schéma de composition clair, répétif et efficace. Trois moments principaux se succèdent, groupés en une seule séquence à l'intérieur du même cadre : le vol magique des sorciers juchés sur des balais ou sur le dos de démons, leur réception dans un lieu isolé et sauvage et enfin le moment clé de l'adoration du bouc satanique.

Fig. 2 - Johannes Tinctoris, Tractatus contra sectam Valdensium, ca 1460. (Ms. Rawlinson D. $410, f^{\circ} 1$. Cliché Bodleian Library, Oxford, UK, D.R.)

Fig. 3 - Johannes Tinctoris, Traité du crisme de Vauderie, ca 1460. (Ms. fr. 961, fo 1. Cliché BnF, Paris, D.R.) 
Fig. 4 - Johannes Tinctoris, Tractatus contra sectam Valdensium, ca 1460. (Ms. 11209, $f^{\circ} 3$. Cliché Bibliothèque royale Albert ler de Bruxelles, D.R.)

7 Plusieurs fidèles, aussi bien des hommes que des femmes, les mains jointes et les genoux fléchis dans le geste de la prière, entourent le diable représenté sous la forme d'un bouc. Les costumes des personnages qui entourent l'animal diabolique signalent un rang social assez élevé. Certains tiennent à la main des cierges allumés tandis que l'un des participants accomplit toujours le baiser obscène sur les parties génitales du bouc. Ainsi une importance particulière est-elle accordée à la scène de l'osculum infame. Ce dernier est le plus souvent perçu comme une inversion du baiser de paix échangé lors du rituel d'engagement vassalique, mais il peut tout aussi bien renvoyer au rite de commixtion qui, dans le cadre de la messe, symbolise la puissance unificatrice de l'Eucharistie. Il est vrai que la présence de cierges allumés entre les mains des adorateurs du diable confère à la scène une étrange solennité liturgique... Bien sûr, audelà d'une indéniable unité de composition, chaque miniature possède ses caractères propres, dus aussi, pour une part, à l'habileté variable des peintres ${ }^{8}$. C'est ainsi que la position de l'animal diffère d'une enluminure à l'autre : dans les manuscrits d'Oxford (fig. 2) et de Paris (fig. 3), le bouc tourne le dos à ses adorateurs, comme pour mieux mettre en évidence la pratique du baiser perverti. Dans la miniature de Bruxelles (fig. 4), en revanche, l'animal satanique se présente de profil, la tête tournée vers le spectateur. En position frontale par rapport à l'observateur, le bouc se détache sur un fond de paysage champêtre baigné d'une douce lumière.

La présence de ces images au seuil du traité de Jean Taincture est d'autant plus surprenante que le texte ne comporte aucune description détaillée du sabbat. Plus doctrinal et éloquent que descriptif, le Traité du crisme de Vauderie ne dit pas grandchose en effet sur la réalité concrète du sabbat. Son auteur se contente d'en effleurer le contenu et renvoie ses lecteurs, pour plus ample information, aux aveux des accusés obtenus par la justice. Du reste, il est clair qu'une forte censure pèse dans ce texte sur cet aspect du crime des sorciers explicitement associé au nefandum, à l'indicible 9 L'image s'établit donc précisément en ce lieu paradoxal où les mots du théologien manquent pour dire l'horreur du crime, en ce lieu où le langage savant se dérobe devant l'indicible.

9 Ne pouvant donc s'appuyer sur le texte qui lui sert de support, l'iconographie du sabbat puise son inspiration ailleurs : dans d'autres traités contemporains ou plus anciens, ou plus simplement dans l'actualité judiciaire. Les grands procès du moment et les révélations de la justice à ce sujet ont pu suffire à suggérer aux miniaturistes cette représentation du sabbat. L'image paraît particulièrement concordante avec les propos que l'inquisiteur d'Arras aurait tenus lors de son sermon général du 9 mai 1460. Il y affirmait en effet que les sorciers et les sorcières trouvaient au sabbat « un diable en forme de bouc, de chien, de singe, et aulcunes fois d'homme, et il l'adoraient et plusieurs d'entre eux lui donnaient leur âme [...] ou du moins quelque chose de leur corps; puis ils baisaient le diable en forme de bouc au derrière, avec chandelles ardentes en leurs mains $»^{10}$. Remarquons cependant que si l'inquisiteur se plait à varier les formes animales sous lesquelles le démon se présente à ses sectateurs (chien, chat), les miniatures, de leur côté, privilégient systématiquement le bouc.

10 Seule la page enluminée du manuscrit de Paris tente, à sa manière, de rendre compte $\mathrm{du}$ caractère protéiforme des manifestations du diable au sabbat: deux médaillons exécutés en camaïeu, pris dans le fin réseau des décorations marginales, reproduisent, 
à plus petite échelle, la scène de l'adoration avec un singe (en bas) et un chat (en haut, à droite). Il n'en reste pas moins que dans l'image principale, c'est le bouc qui campe encore une fois fièrement au milieu de ses sectateurs. La complexe figure du sabbat, que l'on trouve minutieusement décrite dans la littérature d'orientation répressive, se simplifie donc ici pour se cristalliser dans une scène emblématique de l'idolâtrie, ou plus exactement de la démonolâtrie.

11 On aurait tort cependant de n'y voir qu'une simple illustration du péché d'idolâtrie. L'image et les effets spécifiques qu'elle engendre peuvent et doivent nous entraîner plus loin. Cette image du sabbat, en effet, ne représente peut-être pas seulement une étape du rituel d'admission dans la "synagogue » de Satan (le baiser obscène couplé à l'hommage collectif). Elle va plus loin en exprimant, avec ses propres moyens, l'essence même du crime de vauderie, du moins tel que Jean Taincture le concevait lui-même dans son traité : un crime " souverainement mauvais " ${ }^{11}$, portant directement atteinte à la puissance souveraine de Dieu, bref un crime de lèse-majesté divine.

\section{Une violence cachée}

12 L'image, au premier abord, n'exerce aucune violence à l'encontre de l'ordre légitime : en ce sens, elle s'oppose à tout un courant iconographique ultérieur (voir, en particulier, la représentation de la sarabande infernale par Hans Baldung Grien) qui insistera plutôt sur le déchaînement de forces incontrôlées. Beaucoup d'historiens se sont étonnés de cette étrange retenue qui confine à l'immobilité. L'historien espagnol Julio Caro Bajora signalait déjà dans les Sorcières et leur monde que «des miniatures françaises $d u x^{e}$ siècle représentent [...] le sabbat [...] mais les artistes qui en sont les auteurs n'ont pas su lui donner le caractère mi-terrifiant, mi-grotesque que lui donnera Goya en un siècle de totale incrédulité. Ils sont toujours corrects et d'une rare objectivité ${ }^{12}$. Le problème est peut-être plus complexe et plus intéressant. Il se pourrait bien en effet, que sous cette apparente correction se cache, en réalité, une subversion redoutable de l'ordre établi.

13 La tranquillité apparente de la mise en scène n'est pas sans produire un effet d'inquiétante étrangeté. Si l'image induit le trouble, elle le doit surtout à l'ambiguïté de son contenu. Comme bien souvent face à l'image, « on ne sait pas toujours si l'on a bien vu ce qu'il y avait à voir, ni même ce que l'on voit ${ }^{13}$. De fait, cette imagerie cohérente du sabbat, bien loin d'être anecdotique, peut apparaitre comme une citation détournée d'un schéma iconographique majeur dans le répertoire occidental des représentations liturgiques: celui de l'adoration de l'Agneau christique. Ce thème était particulièrement connu dans les Pays-Bas bourguignons en raison de la magistrale interprétation qu'en avaient donné, en 1432, Jan et Hubert Van Eyck dans le fameux retable de Gand dit, justement, de l'Agneau mystique ${ }^{14}$.

Célèbre entre toutes, cette représentation de l'adoration de l'Agneau occupe, à l'intérieur du retable, le panneau central du registre inférieur (fig. 4). Campé sur un autel au centre de la représentation, l'agneau, honoré et protégé par un cercle d'anges, voit converger vers lui l'ensemble des membres de l'Église, toutes générations confondues. Dans le prolongement d'une tradition iconographique déjà ancienne, remontant peut-être à la réforme grégorienne, la scène représente aussi l'Église dans sa dimension à la fois atemporelle et unitaire, comme « corps mystique » du Christ ${ }^{15}$. 
Fig. 5 - Jan et Hubert Van Eyck, L'Agneau mystique, retable de la cathédrale Saint-Bavon, Gand (détail du panneau central). (Extrait de B. Dekeyrer, Les Primitifs flamands, Artois, Bruxelles, 1999, p. 33 , D.R.) liaison étroite avec le péché de luxure ${ }^{19}$. Mais si le bouc renvoie sans doute aux orgies sexuelles qui accompagnent le sabbat, il fonctionne surtout ici comme l'opérateur visuel d'une usurpation. L'image ne bouleverse pas le schéma iconographique traditionnel de l'adoration de l'Agneau, mais elle le subvertit de l'intérieur en mettant une figure à la place d'une autre : le bouc satanique se substitue à l'agneau christique, pourtant seul habilité à occuper cette position souveraine, au centre de l'adoration. Cette image relève ainsi en partie du trompe-l'œil.

Explagneau et le bouc, l'image pousse très loin le jeu de l'équivoque : est-ce vraiment le fait du hasard si, dans la miniature de Paris, de légères touches de blanc viennent réhausser le corps de l'animal diabolique ? La «notion de ressemblance pervertie » avancée par Jérôme Baschet pour caractériser l'évolution de l'image du diable à la fin du Moyen Âge se révèle ici particulièrement pertinente ${ }^{20}$.

19 La figure du bouc comme double de l'Agneau mystique se trouve également renforcée par la résonance eschatologique de la mise en scène. Sous cet aspect, le bouc satanique de l'enluminure de Bruxelles n'est pas sans rappeler la seconde bête de l'Apocalypse qui, dans la vision de Jean, s'oppose à l'agneau. Rappelons seulement que dans le livre de l'Apocalypse, la deuxième "bête » infernale, issue de la "terre ", " avait deux cornes comme un agneau». C'est elle encore qui «séduit les habitants de la terre par les 
prodiges qu'il lui est donné d'accomplir...» et les "incite à dresser une image en l'honneur de la bête $»^{21}$. Le paysage raffiné dont la beauté et le calme apparent contrastent avec la scène terrible de l'adoration du bouc participe de ce trompe-l'œil. L'arbre situé à l'arrière-plan, dans l'axe de symétrie verticale, n'est pas sans évoquer l'«arbre de vie» qui, dans la description de la Jérusalem céleste de l'Apocalypse, se dresse au «milieu de la place de la cité » associé, comme dans notre image, à un «fleuve d'eau vive, brillant comme du cristal qui jaillissait du trône de Dieu et de l'agneau $»^{22}$. Une suite de références et d'allusions facilitent ainsi le glissement d'un champ iconographique à l'autre : le rocher du premier plan pourrait ainsi rappeler le Christ selon une comparaison symbolique usuelle au Moyen Âge ${ }^{23}$. De même, il est évident que le nombre évangélique des douze adorateurs du diable dans la miniature de Paris ne doit rien au hasard...

C'est ici peut-être, dans ce détournement des symboles christiques et des codes de représentation de la majesté positive, que cette image singulière du sabbat rejoint le mieux les idées exprimées dans le Traité du crime de Vauderie. Le théologien de Tournai ne voit, en effet, dans l'essor de la nouvelle secte des sorciers qu'un ultime avatar de la révolte originelle des anges rebelles contre Dieu. Il situe ainsi à l'origine du péril sorcier la volonté usurpatrice du diable de se «rendre semblable au prince souverain $»^{24}$. C'est bien d'ailleurs aussi pour cela que la vauderie relève du crime de majesté. L'enluminure ne ferait ainsi que transposer dans le domaine visuel le projet d'usurpation du "prince des ténèbres». Ici, comme dans d'autres représentations contemporaines du diable, les miniatures permettraient à « la majesté maléfique » de Satan de se déployer avec éclat, menaçante face à celle de Dieu, voire à celle du prince.

\section{Les faux-semblants de la majesté maléfique}

21 Dans ces conditions, dire l'ampleur du crime ne suffisait pas au théologien qu'était avant tout Jean Taincture. Il lui fallait encore consacrer, face au défi satanique, l'unicité absolue de la puissance divine. Il consacre ainsi toute la seconde partie de son traité à démontrer qu'une telle usurpation est impossible car le diable ne peut être tout à fait "semblable » à Dieu. D'une certaine manière, la réflexion de Taincture cherche donc aussi à dissiper les faux-semblants de la majesté diabolique tels que les enluminures précisément les mettent en œuvre.

22 Tout en prenant acte de l'étendue des pouvoirs que la démonologie confère au diable, le théologien s'applique surtout à en préciser les limites : si le diable peut déplacer des corps dans l'espace, il ne peut agir sur le mouvement des étoiles; si le diable peut, à la façon d'un prestidigitateur, faire passer un objet pour un autre, il ne peut en modifier la substance, c'est-à-dire la structure interne. De même encore, si le démon participe lui-même activement à l'orgie sexuelle qui accompagne le sabbat, il n'a pourtant pas la capacité d'engendrer. Le démon en est donc très vite réduit, selon Jean Taincture, à pallier ses propres insuffisances par les vertus de l'illusion.

Il est intéressant de voir que les enluminures elles-mêmes prennent en compte ces limites ou, du moins, jouent avec elles. Cette étrange construction en trompe-l'œil donne ainsi son importance à des écarts de détails. Tels des signaux d'alarme, des anomalies réintroduisent dans l'image une monstruosité diabolique refoulée. À y regarder de plus près, l'imitation de la figure christique reste imparfaite : les délicates 
touches de blanc parfois apposées sur le corps du bouc ne peuvent dissimuler tout à fait la grisaille de son pelage.

Si la miniature de Bruxelles va sans doute le plus loin dans la provocation, il s'agit toujours d'une opération parfaitement maitrisée : plusieurs indices attirent l'attention sur le caractère fallacieux de la mise en scène. Il y a bien sûr, dans le ciel, ces deux démons servant de montures fantastiques aux sorciers. Il y a aussi, entre les pattes du bouc, cette curieuse plante aux feuilles dentelées qui donne d'inquiétantes racines à l'«arbre de vie » situé dans son prolongement vertical. Le choix des couleurs a peutêtre aussi son importance: dans le manuscrit de Bruxelles, la couleur utilisée pour peindre les collines servant de toile de fond à la scène centrale de l'adoration du bouc résulte d'un mélange insolite de jaune et de vert ${ }^{25}$. Il convient certes de rester extrêmement prudent dans un domaine qui ne tolère aucun esprit systématique. Reste que cette combinaison n'en produit pas moins un effet visuel surprenant qui n'est peutêtre pas sans rapport avec l'usage du vert et du jaune dans le langage des couleurs de l'époque. L'association de ces deux tons n'est-elle pas en effet souvent porteuse, dans la symbolique médiévale des couleurs, d'une idée de désordre ou de folie ${ }^{26}$ ? On peut également constater que si la figure monumentale du bouc, les quatre pattes solidement posées sur le sol, imite parfaitement la posture de l'Agneau mystique du retable de Gand, sa position (tête à droite) est inversée par rapport à celle de l'animal christique. Autre détail significatif : les trois enluminures relèguent les figures les plus insolites (démons hideux) ou les plus controversées (sorcières chevauchant des balais) dans la zone céleste, là où précisément le diable, selon Jean Taincture, fabrique à partir de la condensation de l'air ses «illusions fantastiques». Dans l'enluminure du manuscrit de Paris, le ciel fait d'ailleurs l'objet d'un traitement singulier qui souligne l'opacité de la matière dans laquelle évoluent ces figures monstrueuses: un filtre de grisaille, dont l'épaisseur est encore renforcée par des stries dorées, atténue fortement l'éclat de la couleur bleue utilisée initialement pour peindre le ciel. Dans le coin supérieur gauche, une déchirure dans les nuages laisse d'ailleurs apparaître, outre le bleu d'origine, l'éclat lumineux de la lune. La représentation du ciel semble ainsi faire écho aux doctes sentences de Jean Taincture relatives aux manipulations diaboliques des corps célestes: "les étoiles du firmament ne peuvent être privées de leur belle lumière ». Cela dit, de "grosses nuées peuvent nous dissimuler la clarté de leur rayonnement", entrainant dès lors une "mutacion es étoilles mais seulement en l'air $»^{27}$.

D'une manière générale, la vignette centrale de la page enluminée du manuscrit de Paris se signale par la tonalité sombre de ses couleurs qui contraste fortement avec l'éclatant coloris des marges. Elle partage, du reste, ce trait d'originalité avec la miniature ornant le manuscrit de Bruxelles. Il faut savoir en effet que la critique hésite toujours, en dépit de traits stylistiques concordants, à attribuer cette dernière miniature à l'atelier de Loyset Liédet, en raison - nous dit-on- d'un "coloris » excessivement "modéré $»^{28}$. On peut certes trouver à cet affadissement des couleurs une explication de type « réaliste » en arguant du caractère nocturne de la scène. On ne peut cependant s'empêcher de mettre en rapport cette trouble opacité de la lumière entourant l'adoration du bouc avec l'usage et la signification de la transparence dans la peinture religieuse de l'école du Nord. On se plait ainsi volontiers, à propos du retable des frères Van Eyck, à souligner la qualité particulière, presque cristalline, de la lumière dans laquelle baigne la scène de l'adoration de l'Agneau mystique. À l'inverse, 
il est permis de penser que l'épaisseur trouble de l'atmosphère dans laquelle évoluent les sorcières signale aussi la présence du mal ${ }^{29}$.

Si des indices de ce type trahissent la nature aberrante des prétentions diaboliques, une telle image n'en garde pas moins une redoutable efficacité visuelle. L'enluminurefrontispice partage avec l'illusion diabolique ce pouvoir, qui est celui de l'image, de donner force de présence à ce mystérieux et inquiétant « corps mystique » du diable...

C'est pourquoi, sans doute, une image aussi dérangeante ne peut guère se concevoir en dehors de son environnement écrit et savant. La présence dans le Traité du crisme de Vauderie d'une telle image du sabbat trouve sa légitimité dans son rapport étroit à un texte qui en neutralise, pour une part, les charmes délétères. Ce contrôle du texte sur l'image peut prendre un caractère immédiat, comme dans le manuscrit de Bruxelles, où l'incipit, placée au bas de la page enluminée, vient clairement modifier et éclairer le sens négatif de l'image : «Par l'envie du dyable la mort print entrée ou monde. Et ce le ensuivent ceulx qui tiennent son parti ».

Mais plus fondamentalement, cette représentation du sabbat n'est pas sans relation avec toute la réflexion que Jean Taincture consacre, dans la seconde moitié de son traité, à la dénonciation des malversations diaboliques. Dès l'introduction, l'auteur, en présentant le projet de cette partie plus spéculative de son ouvrage, adressait ainsi une mise en garde à ceux qui « s'arrestent es ymages et semblances des choses comme es vrayes choses $»^{30}$.

\section{Une riposte théologique}

Compte tenu de la puissance suggestive de l'illusion, il était vital pour Jean Taincture de briser le miroir des apparences. C'est donc par-delà le visible lui-même que le théologien de Tournai s'emploie à découvrir la faille cachée susceptible de réduire à néant les prétentions du diable à se «rendre semblable " à Dieu, c'est-à-dire aussi à usurper la place du Christ. Si Jean Taincture dénonce l'illusion fausse, c'est qu'elle enferme le regard dans l'horizon du sensible en se substituant à la création divine. Le rôle du discours savant et démonologique est alors de dévoiler la logique usurpatrice du simulacre et de manifester, par la force du savoir, la différence irréductible entre la pâle copie et son modèle.

Toute la réflexion de Taincture s'organise autour de l'idée que seul le Christ, à travers le mystère de l'Incarnation, peut prétendre représenter Dieu, en être véritablement l'image - étant entendu que pour la tradition théologique médiévale, il ne peut y avoir d'image proprement dite que s'il existe entre la copie et son modèle un rapport d'origine, ce que Thomas d'Aquin, à la suite d'Aristote, appelle également un lien de similitude $^{31}$. Or, la similitude, bien au-delà de la simple ressemblance trait pour trait, implique, entre l'image et son modèle, une identité de nature, un lien d'ordre généalogique. À l'insupportable prétention du diable d'égaler le Dieu souverain, Jean Taincture réplique ainsi avec force, et en termes totalement thomistes, qu'il faut que "le semblable soit engendré de son semblable ${ }^{32}$. On comprend peut-être mieux dès lors pourquoi le théologien de Tournai, fin connaisseur de l'œuvre de Thomas d'Aquin, tenait tant à rappeler que le démon ne pouvait rien créer ex nihilo, qu'il ne pouvait surtout forcer les voies de l'engendrement pour donner la vie. 
31 Dans cette perspective, la mise en scène du pseudo-corps mystique du diable n'est qu'une parodie de la présence réelle où le démon échoue à reproduire le miracle eucharistique. Disqualifiée par son déficit de chair et par ses propres origines créées, la puissance diabolique ne peut ainsi aboutir, selon Taincture, qu'à une caricature imparfaite du corps du Christ. La puissance démoniaque, dans les limites que lui assigne le théologien, se révèle incapable de dépasser le stade de l'image vide, du simulacre. L'illusion diabolique n'est qu'une figure creuse à laquelle manque fondamentalement la "substance de vie " $33^{3}$. À la manière des figures cabalistiques tracées par les magiciens qui « ne sont d'aucunes efficace à causer reaulx effects ne chose naturelle $»^{34}$, l'illusion diabolique n'existe donc que par son contour, que par les «traits de lignes» qui la circonscrivent. En démasquant l'imposture diabolique, Jean Taincture en vient à rétablir, par delà les seules apparences sensibles, les conditions légitimes d'avènement de la seule image de Dieu possible : celle du Fils, image filiale du père, «engendré et non pas créé ».

Soustraire le domaine de la vie à l'emprise diabolique, verrouiller les accès à l'engendrement et au processus généalogique de l'Incarnation seraient ainsi, pour Jean Taincture, autant de moyens de protéger le corps du Christ, image du père, contre toutes les manipulations subversives du diable. Y compris et peut-être surtout lorsque celles-ci empruntent les séductions de l'art.

\section{NOTES}

1. Paris, BnF, ms. 12476. L'explicit nous apprend, en effet, que le texte fut « escript ou cloistre de l'église Nostre Dame d'Arras, en l'an de l'Incarnacion de Nostre Seigneur 1451 ». Sur ce manuscrit et les conditions de sa réalisation, voir F. Avril, "Le Maître du Missel de Paul Beye », dans A. NOTTER, Fragments d'une splendeur. Arras à la fin du Moyen Âge, Tourcoing, 2000, p. 49-53. Ce manuscrit a aussi la particularité de comporter, au fo $105 \mathrm{v}^{\circ}$, l'une des premières représentations connues de sorcières montées sur des bâtons ou des balais. Voir l'étude très précise que leur consacrent M. OSTORERO et J.-C. S CHMITT, «Le balai des sorcières. Note sur une illustration marginale du manuscrit Paris, BnF, ms. fr. $12476, \mathrm{f}^{\circ} 105 \mathrm{v}^{\circ}$ ", dans M. OSTORERO et alii, L'Imaginaire du sabbat. Édition critique des textes les plus anciens (1430 ca-1440 ca), Lausanne, 1999, p. 501-508.

2. Paris, BnF, ms. fr. $961, \mathrm{f}^{\circ} 1 \mathrm{r}^{\circ}$; Bruxelles, Bibliothèque royale, ms. $11209, \mathrm{f}^{\circ} 3 \mathrm{r}^{\circ}$; Oxford, Bodleian Library, ms. Rawl. D. 410, $\mathrm{f}^{\circ} 1 \mathrm{r}^{\circ}$. Elles font toutes trois l'objet d'une courte présentation technique dans M. PREAUD, Les Sorcières, Paris, 1973, $\mathrm{n}^{\text {os }} 87,88,89$, p. 58-60. Voir également E. BALMAS, «Il traité de Vauderie di Johannes Tinctor », Protestantesimo, 1, 1979, p. 1-26.

3. Voir G. DOGAER, Flemish miniature painting in the Fifteenth and Sixteenth centuries, Amsterdam, 1987, p. 107-112. Le manuscrit de Bruxelles figurait déjà dans la bibliothèque ducale en 1467 comme en témoigne l'inventaire dressé à cette date. Il s'agit probablement d'une œuvre conçue pour le commerce et entrée dans les collections de Philippe le Bon par achat.

4. Paris, BnF, ms. fr. 961. Cette copie fut réalisée à Bruges, vers 1470, pour le compte de Louis de Bruges, seigneur de la Gruthuse. Elle contient une miniature attribuée à l'atelier de Philippe de Mazerolles $\left(\mathrm{f}^{\circ} 5 \mathrm{r}^{\circ}\right)$. Voir J. B. B. VAN PRAET, Recherches sur Louis de Bruges, seigneur de la Gruuthuse, suivies de la notice des manuscrits qui lui ont appartenu, et dont la plus grande partie se conserve à la 
bibliothèque du roi, Paris, 1831, p. 122-123 ; et plus récemment, M. P. J. Martens dir., Lodewijk van Gruuthuse. Mecenas en Europees diplomaat, ca 1427-1492, Bruges, 1992. Comme on l'a vu, un autre exemplaire de luxe de ce traité est conservé à Oxford. D'origine indéterminée - bien qu'elle ne puisse être que flamande - cette version est également incomplète. Elle ne comporte en effet ni prologue ni index. Voir Catalogi codicum manuscriptorum Bibliotecae Bodleianae, V, 3, Oxford, 1893, col. 298-299. Il est possible que ce texte soit parvenu outre-Manche par l'entremise de Louis de Bruges. Ce grand officier de l'État bourguignon entretenait en effet des relations privilégiées avec l'Angleterre: il y effectua notamment plusieurs missions diplomatiques pour le compte de Philippe le Bon (1460-1461 et 1465-1467). Ayant fastueusement reçu à Bruges le roi Édouard IV, lors de son exil forcé sur le continent en 1470 , ce dernier lui témoigna plus tard sa reconnaissance en le faisant comte de Winchester (1472). Voir M. VALE, « An Anglo-Burgundian nobleman and art patron : Louis de Bruges, Lord of la Gruthuyse and Earl of Winchester ", dans C. BARRON et N. SAUL dir., England and the Low Countries in the Late Middle Ages, New-York, 1995, p. $115-131$.

5. Le traité de J. Taincture est désormais disponible dans une édition moderne grâce à E. VAN BALBERGHE et F. DUVAL, Jean Tinctor, Invectives contre la secte de vauderie, Tournai/Louvain-laNeuve, 1999. Toutes nos références renvoient au ms. fr. 961 de la BnF qui a servi de base à cette édition. Sur le texte lui-même, voir également E. VAN B ALBERGHE et J.-F. GILMONT, «Les Théologiens et la Vauderie au $\mathrm{XV}^{\mathrm{e}}$ s.», Miscellanea codicologica, F. Masai dicata, Gand, 1979, p. 393-411; F. DUVAL, "Jean Tinctor, auteur et traducteur des Invectives contre la secte de vauderie ", Romania, t. 117, 1999, p. 186-217.

6. Sur le parcours et l'œuvre universitaire de J. Taincture, voir M. GRABMANN, « Der Belgische Thomist Johannes Tinctoris und die Enstehung des Kommentars zur Summa Theologiae des hl. Thomas von Aquin ", Studia mediaevalia in honorem RP Raymundi Joseph Martin OP, Bruges, 1948, p. 409-436 ; repris avec additions dans Mittelalterliches Geistesleben, t. 3, 1956, p. 411-432.

7. Sur la Vauderie d'Arras, voir l'ouvrage pionnier mais toujours indispensable d'A. DUVERGER, Le Premier Grand procès de sorcellerie aux Pays-Bas : la Vauderie dans les États de Philippe le Bon, Arras, 1885. Voir aussi G. A. SINGER, La Vauderie d'Arras 1459-1491: an episode of witchcraft in later medieval France, Diss. Univ. of Maryland, 1975 et plus récemment G. GONNET, «La Vauderie d'Arras », I Valdesi e l'Europa, Collana della Società di Studi Valdesi, 9, Torre de Pelice, 1982, p. 99-113. Nous nous permettons de renvoyer à notre thèse de doctorat, F. MERCIER, La Vauderie d'Arras (1459-1491) ou l'émergence contrariée d'une nouvelle souveraineté autour des ducs Valois de Bourgogne ( $\mathrm{x} v^{e} \mathrm{~s}$.), 3 vol., Université de Lyon II, 2001.

8. La miniature ornant le manuscrit d'Oxford, d'une qualité picturale médiocre, souffre en effet de la comparaison avec celles de Paris et Bruxelles. Voir O. PÄcht, J. J. G. ALEXANDER, Illuminated Manuscripts in the Bodleian Library Oxford, Oxford, 1966, t. 1, n 379, p. 29, pl. XXX.

9. Dans la version latine du traité de Taincture, les occurrences précises du terme nefandum et de ses dérivés se cristallisent notamment autour des turpitudes sexuelles contre-nature imputées aux vaudois-sorciers mais aussi, et de manière plus importante encore, autour de la profanation de l'hostie (voir ms. Bruxelles, lat. 11449-51, fos $36 \mathrm{v}^{\circ}-37 \mathrm{r}^{\circ}$ ). Le «noyau dur " des activités criminelles de la secte est même censuré dans le texte français comme pour mieux souligner le poids de l'indicible. Sur les rapports étroits qui s'établissent à la fin du Moyen Âge entre la figure de l'indicible et les crimes de lèse-majesté, voir J. CHIFFOLEAU, « Dire l'indicible : remarques sur la catégorie du nefandum du XII ${ }^{\mathrm{e}}$ au XII' ${ }^{\mathrm{e}}$ s. ", Annales ESC 45, mars-avril 1990, p. 289-324.

10. J. Du CLERCQ, Mémoires, F. DE REIFFENBERG éd., Bruxelles, 1835-36, L. IV, chap. 4, p. 21.

11. J. TAINCTURE, op. cit., fo $19 \mathrm{r}^{\circ}$ : « ...vrayement cestui crime est souverainement mauvais (c'est moi qui souligne) qui prent son commencement en apostasie et mespris de la religion crestienne ».

12. J. C. B AROJA, Les Sorcières et leur monde, (1961) trad. fr., Paris, 1972, p. 109. Robert Muchembled rejoint ce jugement en notant, à propos de ces miniatures, que «l'ensemble n'est 
pas réellement effrayant, plutôt curieux, anecdotique "; R. MUCHEMBLED, Une histoire du diable, $X I I^{e}-X X^{e}$ s., Paris, 2000, p. 63.

13. J. B ASCHET, «Inventivité et sérialité des images médiévales. Pour une approche iconographique élargie », Annales HSS, janvier-février 1996, 1, p. 93-133, p. 104.

14. Sur ce fameux retable, voir surtout E. Panofsky, Les Primitifs flamands, (1971), trad. fr., Paris, 1992, p. 373-435 et E. DHANENS, Van Eyck, Paris, 1980, p. 72-121.

15. Voir H. TOUBERT, «Les Représentations de l'Ecclesia dans l'art des $\mathrm{X}^{\mathrm{e}}-\mathrm{XII}{ }^{\mathrm{e}} \mathrm{s}$. ", Un art dirigé. Réforme grégorienne et iconographie, Paris, 1990, p. 37-67. Il se pourrait d'ailleurs que le retable de Gand soit né au départ d'une commande de l'évêque de Luxembourg désireux de célébrer la résolution du Grand Schisme à travers une vision grandiose de l'unité de l'Église. Cette hypothèse se trouve au centre de l'étude d'A. M. ARMAND, L'Agneau mystique. Ex voto national de la Belgique chrétienne. "De la foi victorieuse ». Essai d'interprétation symbolique, Paris, 1961. Sur la complexité de la genèse du retable, voir E. PANOFSKY, «L'Annonciation Friedsam et le problème du retable de Gand », Peinture et dévotion en Europe du Nord à la fin du Moyen Âge, Paris, 1997, p. 29-46. Il est clair, d'autre part, que le thème de l'Adoration de l'agneau, toujours étroitement lié à l'Apocalypse, s'enracine dans une longue tradition iconographique remontant à l'époque paléochrétienne. Voir à ce sujet F. NICOLASH, Das Lamm als Christussymbol, Vienne, 1963 et J. WIRTH, L'Image médiévale, Paris, 1989, p. 146-152.

16. Les Heures de Turin-Milan (détruites dans l'incendie de la Bibliothèque royale de Turin en 1904) comportaient effectivement deux séries de miniatures de caractère visiblement eyckien (il s'agirait d'œuvres de jeunesse). L'une d'elles représentant la «Virgo inter virgines » serait directement issue de la "Cohorte des vierges adorant l'Agneau». Voir P. DURRIEU, Heures de Turin, Paris, 1902, pl. XXXVI et E. PANOFSKY, Les Primitifs flamands, op. cit., p. 411-416, fig. 304.

17. Sur les valeurs positives de l'immobilité dans l'image médiévale, voir J.-C. SCHMITT, La Raison des gestes dans l'Occident médiéval, Paris, 1990, p. 28-31.

18. Sur l'importance des jeux de regard entre les démons et les hommes, voir J. WIRTH, «La Démonologie de Bosch », dans Diables et diableries. La représentation du diable dans la gravure des $\mathrm{XV}^{e}$ et XVI ${ }^{e}$ s., Genève, 1976, p. 71-85.

19. M. PRÉAUD, op. cit., p. 69.

20. J. BASCHET, «Satan ou la majesté maléfique dans les miniatures à la fin du Moyen Âge », N. NABERT dir., Le Mal et le diable. Leurs figures à la fin du Moyen Âge, Paris, 1996, p. 187-210. Voir plus particulièrement p. 199.

21. Apocalypse, XIII, 11-18.

22. Apocalypse, XXII, 1-5.

23. Sur la figure de la pierre comme élément caractéristique du «décor christique " dans la peinture médiévale, voir G. DIDI-HUBERMAN, Fra Angelico. Dissemblance et figuration, Paris, 1990, particulièrement p. 61-62.

24. Dans le court prologue sur lequel s'ouvre son traité, Jean Taincture retrace en effet les circonstances de la révolte des anges rebelles contre Dieu. À l'origine de cette révolte se trouverait la volonté de l'ange Lucifer de «se rendre semblable au prince souverain ", op. cit., fo $7 \mathrm{v}^{\circ}$.

25. Ajoutons ici que se pose un problème de restitution des couleurs originales par la reproduction. En l'occurrence, cette couleur spécifique passe assez mal à la reprographie qui tend à l'éclaircir au profit du jaune.

26. Voir M. PASTOUREAU, «Formes et couleurs du désordre : le jaune avec le vert ", dans Figures et couleurs. Études sur la symbolique et la sensibilité médiévales, Paris, 1996, p. 23-36.

27. J. TAINCTURE, op. cit., fo $97 \mathrm{v}^{\circ}$.

28. Pour reprendre l'expression des auteurs du catalogue de l'exposition «organisée à $l^{\prime}$ occasion du $400^{\mathrm{e}}$ anniversaire de la fondation de la Bibliothèque royale de Philippe II, le 12 avril 1559 » et consacrée à la miniature flamande: «La miniature a été attribuée à Liédet, mais de 
quelle période de sa production pourrait provenir un tableau d'un coloris si modéré ?». Voir L. M. J. Delaissé dir., La Miniature flamande. Le mécénat de Philippe le Bon, Bruxelles, 1959, notice $\mathrm{n}^{\circ} 113$, p. 110.

29. L'opposition entre l'opacité trouble des illusions diaboliques et la clarté limpide des images divines se trouve déjà dans saint Augustin, De civitate Dei, X, 12. Sur ce point, voir J.Céard, "Démons et merveilles dans la basse latinité : l'héritage de saint Augustin », Démons et merveilles au Moyen Âge, Nice, 1990, p. 25-38, spécialement p. 33-36.

30. J. TAINCTURE, op. cit., fo $18 \mathrm{v}^{\circ}$.

31. Ce qui fera dire à Thomas d'Aquin «qu'un œuf ne peut être l'image d'un autre œuf », car il ne dérive pas de lui. Sur cette question de l'identité de nature à la base de la définition théologique de l'image, voir A.BESANÇON, L'Image interdite, une histoire intellectuelle de l'iconoclasme, Paris, 1994, p. 224. Sur la conception thomiste de l'image, voir encore J. WIRTH, "Structure et fonctions de l'image chez saint Thomas d'Aquin ", L'Image, fonctions et usages des images dans l'Occident médiéval, Cahiers du Léopard d'or, 5, Paris, 1996, p. 39-57.

32. J. TAINCTURE, op. cit., fo $98 \mathrm{r}^{\circ}$ : «Il n'est, dis-je, une espèce estre transmuée en autre. Car comme selon les philosophes une chose ne puist estre faicte de chascune autre, mais faille que par naturele transmutacion chascune chose se face de certaine et déterminée matière et que le semblable soit engendré de son semblable ».

33. J. TAINCTURE, op. cit., fos $100 \mathrm{r}^{\circ}-101 \mathrm{r}^{\circ}$ : «Une substance de tous poins séparée du corps et qui est subsistante en la pureté immatérielle sans quelque deppendence de chose corporelle, comme est l'angele, ne puet vivifier substanciellement un corps. Et comme la seconde et accidentele perfection présuppose nécessairement la perfection première et essencielle, il s'ensieult que opéracion de vie ne puet estre en ung corps sans la substance de vie. Et pourtant, les angeles ne pevent en corps non vivifiés exercer opéracion de vie. Ainsi toutes les euvres et opéracions semblans estre euvres de vie qu'ils font es corps qu'ils ont prins, ne sont pas vrayes, mais seulement apparans ».

34. J. TAINCTURE, op. cit., fo $84 \mathrm{v}^{\circ}$.

\section{RÉSUMÉS}

Les représentations figurées du sabbat des sorcières sont rares avant la fin du Moyen Âge. Trois miniatures flamandes placées en frontispice de plusieurs manuscrits français enluminés du Traité du crisme de Vauderie du théologien Jean Taincture constituent une remarquable exception. Datées des années 1460-1470, elles donnent à voir une scène d'adoration du diable sous la forme d'un bouc. Une interprétation hâtive en a fait l'expression exclusive de la luxure. Rapprochées du thème iconographique de l'Agneau mystique, tel qu'il avait été élaboré par Van Eyck autour de 1430 , et surtout du texte de J. Taincture, ces images prennent une nouvelle signification. À sa manière, l'imagerie flamande du sabbat rend compte de ce que le crime de sorcellerie est devenu $\mathrm{au} \mathrm{XV}$ siècle : une atteinte fondamentale à la toute-puissance divine.

Illustrated representations of the witches' sabbath are rare before the end of the Middle Ages. Three Flemish miniatures forming the frontispiece of several French illustrated manuscripts of the Traité de crisme de Vauderie by the theologian Jean Taincture constitute a remarkable exception. Dating from 1460-1470, they show a scene of worship of the devil as Goat and have been interpreted precipitately as purely an expression of lust. However, if these images are seen 
in the context of the iconographic theme of the Mystical Lamb, as elaborated by Van Eyck towards 1430, and more especially of the Jean Taincture's text, they assume a new meaning. In its own way, the Flemish imagery of the witches' sabbath demonstrates what the crime of witchcraft had become in the 15th century: a serious undermining of divine omnipotence.

INDEX

Keywords : 15 th century, Burgundy, devil worship, image, mystical lamb

Mots-clés : adoration du diable, agneau mystique, Bourgogne, image, xve siècle

\section{AUTEUR}

\section{FRANCK MERCIER}

Université de Rennes II - Haute-Bretagne, Département d'Histoire, UFR de Sciences Sociales, 6, av. Gaston Berger, 35043 Rennes Cedex 